\title{
The Partial Derivative Machine
}

\author{
Grant Sherer, Mary Bridget Kustusch, Corinne A. Manogue and David Roundy
}

\author{
Department of Physics, Weniger 301, Oregon State University, Corvallis, Oregon 97331
}

\begin{abstract}
Research has shown that students struggle to understand the use of partial derivatives in thermodynamics. We have designed an apparatus, which we have called a Partial Derivative Machine, that serves as a mechanical analogue of a thermodynamic system. Using this device, students have a tangible way to wrestle with issues related to partial derivatives and thermodynamics, such as which variables are held fixed, how many variables are independent, and how energy can be added to a system. In this paper, we present a description of the apparatus, an introduction to the associated activities, and an overview of how this apparatus can be connected to thermodynamic systems.
\end{abstract}

Keywords: Upper-Division Physics, Physics Education, Partial Derivatives, Thermodynamics PACS: 01.40.Fk, 01.40.gb, 05.70.-a

\section{MOTIVATION}

Students often struggle with thermodynamics at least in part due to the unfamiliarity and complexity of partial derivatives [1-6]. Despite the development of new representations and activities designed to help students master the use of partial derivatives, they continue to struggle when faced with unfamiliar mathematical manipulations with new physical quantities such as entropy. Additionally, students have been taught in their mathematics courses, and sometimes in earlier physics courses, that when taking a partial derivative, "everything else" is held constant - an operational definition that is particularly problematic in thermodynamics.

During the 2012-2013 academic year, we designed an apparatus, which we have called a Partial Derivative Machine (PDM), with the goal of introducing partial derivatives in a physical context that is familiar to students through a mechanical analogue of a thermodynamic system. This apparatus, along with the activities described in this paper, represent a small piece of an extensive redesign of Energy and Entropy, which is the thermodynamics portion of the Paradigms in Physics sequence at Oregon State University [7]. The Paradigms in Physics Project is an on-going and comprehensive reform of the upper-division physics curriculum [8,9]. Most of the changes to Energy and Entropy have involved the development of activities and sequences designed to provide more physical significance to thermodynamics through concrete examples. In particular, we view kinesthetic experiences and experiments (both laboratory and thought) as valuable representations for students to experience $[6,10]$. Descriptions of some of these activities can be found in other recent publications $[6,11,12]$. The PDM is the most recent example of this kind of reform and was designed for use in the Interlude, a one week session (7 contact hours) at the beginning of Energy and Entropy

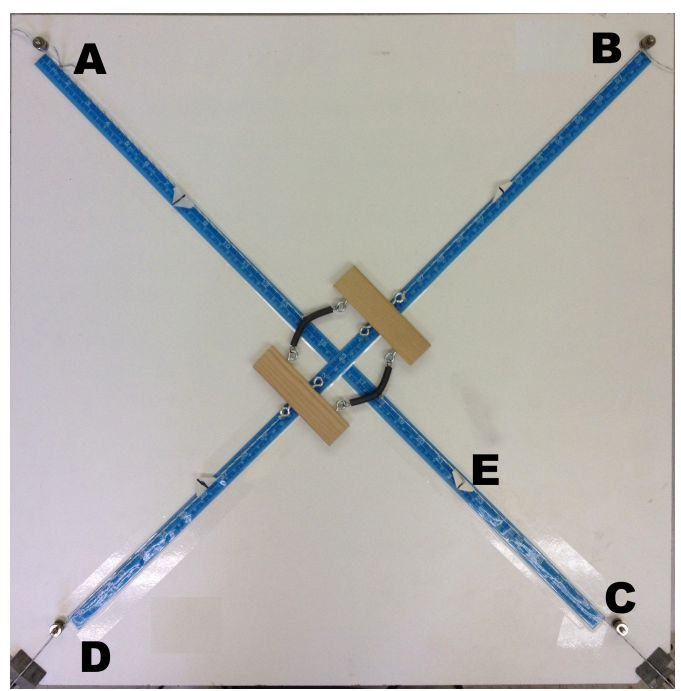

FIGURE 1. The PDM, (A/B) Corners with only knob, (C/D) Corners with pulley and knob, (E) Measuring Flag.

that covers mathematical techniques relevant to thermodynamics [7]. The Partial Derivative Machine was first used in the Spring 2013 Interlude, a class of roughly 30 students.

\section{THE PARTIAL DERIVATIVE MACHINE}

The Partial Derivative Machine is an apparatus consisting of a central spring system that can be stretched via four strings extending outward from the center (See Fig. 1). Alternative central systems can be used for this activity including a loop of string, a piece of spandex, and other combinations of springs (see Fig. 2). This central system is on a large piece of particle board which features a pulley on two adjacent corners (Corners $\mathrm{C}$ and 


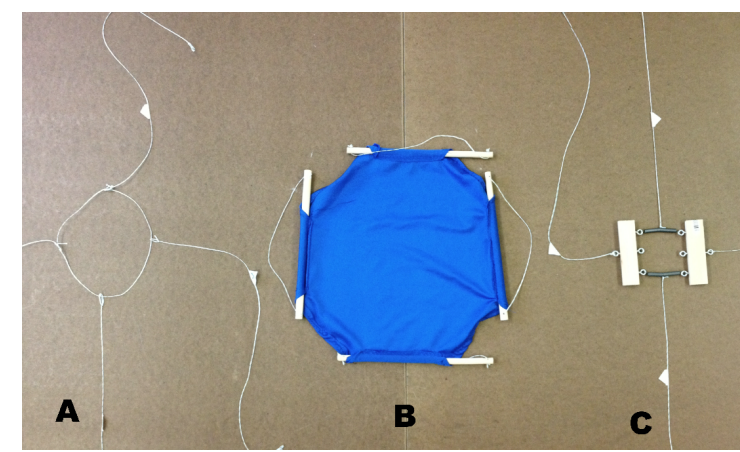

FIGURE 2. Example Central Systems: (A) Loop of String, (B) Piece of Spandex, and (C) Spring System.

D in Fig. 1), and a knob on all four corners (See Fig. 3). By tightening the knobs at A and B (See Fig. 1), one can hold the system in place while adding weights to the hanging strings, allowing one to manipulate the state of the system.

In order to more easily measure the stretching of the system, a measuring tape is placed on the board parallel to each string and flags are added to the strings (Example labeled E in Fig. 1). By labeling the axis from corner B to corner D as the "X-axis" and the axis from corner A to corner $\mathrm{C}$ as the "Y-axis" the instructor is able to define four quantities for this experiment:

1. $x$, the distance between the flags on the $\mathrm{X}$ strings

2. $y$, the distance between the flags on the Y strings

3. $F_{x}$, the tension in the $\mathrm{X}$ oriented strings

4. $F_{y}$, the tension in the $\mathrm{Y}$ oriented strings

There are two conditions under which the system can be manipulated. The first method involves tightening the knob on corner $\mathrm{C}$ or $\mathrm{D}$ to pin a third string, thereby fixing $x$ or $y$, and then increasing the mass on the freely hanging string. For example, pinning the knob at $\mathrm{C}$ would fix $y$, then adding weight to the $\mathrm{X}$ string would increase $x, F_{x}$, and $F_{y}$.

Alternatively, one can leave both the $\mathrm{X}$ and $\mathrm{Y}$ strings free and add weights to one or both. In doing so, placing weight on a string causes the system to stretch in one direction while compressing in the other direction as the system balances the forces. For example, adding weight to the $\mathrm{X}$ string would cause $x$ and $F_{x}$ to increase while $F_{y}$ stays constant and $y$ decreases.

It is important to note that as weights are added it is not uncommon for the system to shift from its centered position. In order to keep $F_{x}$ and $F_{y}$ orthogonal as the system is shifted, students are told to temporarily loosen knobs at A and B to recenter the system between measurements. If done correctly, this action is only a translation of the system. Thus it does not result in stretching or

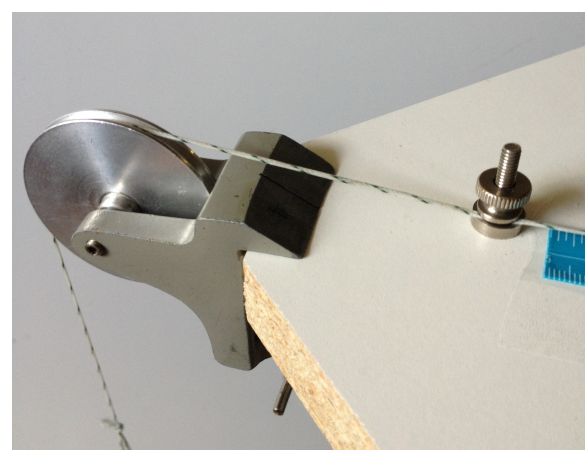

FIGURE 3. Corner with Pulley and Knob (C/D in Fig. 1).

compressing the system and does not impact any of the measurements students are instructed to make.

\section{PLAYING WITH THE MACHINE}

When first introduced to the Partial Derivative Machines, the central system was hidden from students through the use of a "black box" (See Fig. 4). With only the knowledge that there were four strings extending from this box, students were asked to determine:

- The properties of the system that can be controlled.

- The properties of the system that can be measured.

- The number of independent properties of the system.

Students worked briefly in groups of 3 students to answer this prompt and then were brought back together for a class discussion. During the wrap-up discussion, students listed a number of controllable properties including the position of the central system relative to the center of the board, the forces applied to the system, and the amount the system was stretched in either direction. Students decided it was possible to measure $x$ and $y$ by taking values for the positions of the flags, and to measure $F_{x}$ and $F_{y}$ by noting the mass hung from the relevant string.

Many students did not realize however that the tension in a particular string is not equivalent to the weight hung from that string if the corresponding knob is locked since the mass becomes irrelevant when the string is pinned down. Most students also determined that only two of these properties could be controlled independently and that manipulating a pair of parameters caused a responsive change in the other parameters.

\section{FINDING A SIMPLE DERIVATIVE}

Once students were familiar with the machine, they were asked in a second exercise to find $\frac{\partial x}{\partial F_{x}}$ and had to con- 


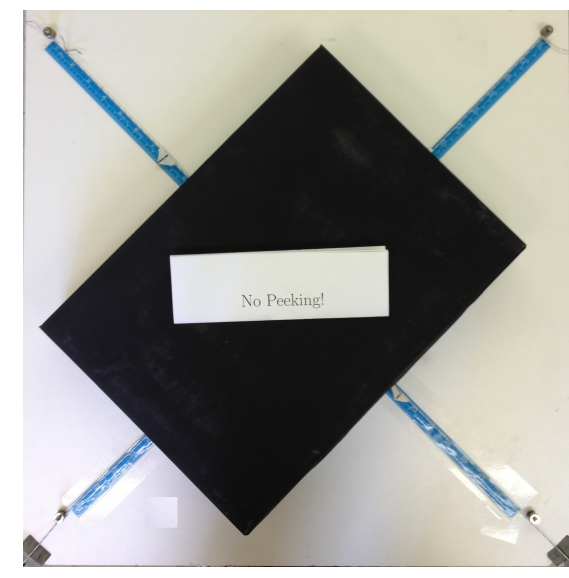

FIGURE 4. PDM with "Black Box" included.

sider that there were two possible options: $\left(\frac{\partial x}{\partial F_{x}}\right)_{y}$ and $\left(\frac{\partial x}{\partial F_{x}}\right)_{F_{y}}$. As an introduction, the instructor defined the concept of stretchability as it relates to the system and distinguished between the "isowidth" (constant $y$ ) and "isoforce" (constant $F_{y}$ ) stretchabilities.

After collecting data sets, plotting results, and calculating numerical values for both quantities students were asked to present their results to the class. The focus of the presentation was not to provide the class with numerical values, but to explain the techniques used to both measure and calculate the necessary information. The approach of some groups was to take a few measurements of the form $\left(F_{x}, x\right)$ and approximate the derivative with the quantity $\frac{\Delta x}{\Delta F_{x}}$. Other groups chose instead to plot $x$ as a function of $F_{x}$.

Due to the different systems under the black boxes, the numerical values for the "isowidth" stretchability and "isoforce" stretchability varied widely from group to group. The relationship between $x$ and $F_{x}$ also varied from system to system - some groups found a linear relationship while others found that the plot was clearly nonlinear.

After these presentations and discussion of the results, students removed the "black box" to see the central systems. Students then walked around the classroom observing other groups' systems to see how each apparatus was different. This allowed for discussion of why particular systems behaved as they did and why particular variables were dependent or independent of each other for each system.

These observations were followed by a whole-class discussion. The instructor asked students to consider if this activity was consistent with or contradicted the idea that one takes a partial derivative while holding "everything else" constant. Next, the class revisited the number of independent variables and which could be set simulta- neously.

It was not obvious to some students that $y$ and $F_{y}$ were relevant quantities when changing $x$ and $F_{x}$. To address this concern the instructor conducted a demonstration making use of the piece of spandex (B in Fig. 2). Having one student grab a pair of opposite handles and hold them a fixed distance apart, a second student was instructed to stretch the spandex in the other direction, which simulated measuring $\left(\frac{\partial x}{\partial F_{x}}\right)_{y}$. It then became abundantly clear to the first student that in order to maintain a constant $y$ it was necessary for $F_{y}$ to increase as $F_{x}$ increased. We have repeatedly found that the kinesthetic effect of feeling the force increase in this demonstration helps people notice that the force and displacement in the two directions are coupled.

\section{INTEGRATED LAB}

As a preface to a major activity associated with the Partial Derivative Machines, students were given a review lecture on:

- Calculating changes in potential energy, $\Delta U$, as the work, $W$, done on the system

- Finding potential energy of stretched springs

- Work as the integral of force, $W=\int F d x$

After this review they proceeded to conduct a laboratory experiment. The primary task was to measure the potential energy stored in the spring system of the Partial Derivative Machine, however a process to determine this function was not explicitly given. The review of work, potential energy, and springs prior to data collection was designed to help students make the connection that the potential energy could be obtained from the work done on the system. Since the system was now two dimensional, using $W=\int \vec{F} \cdot d \vec{r}$ required finding the work done on the system in both the $\mathrm{X}$ and $\mathrm{Y}$ directions.

One possible solution method that determines all necessary information is:

1. Starting at a particular $x=x_{o}$, where $\Delta x=0$, take measurements of $y$ while changing $F_{y}$ in uniform steps, e.g., $0.05 \mathrm{~kg} \times 9.81 \mathrm{~m} / \mathrm{s}^{2}$.

2. Set subsequent $x$ values by loosening knob $\mathrm{D}$, incrementing $F_{x}$ by small uniform steps, and then tightening knob D.

3. Repeat step 1 for each new fixed $x$ value.

4. Using the data and numerical integration of $F_{x} d x$ and $F_{y} d y$, approximate the value of $U(x, y)$.

This process gave students the data needed to get from any state $\left(x_{1}, y_{1}\right)$ to a different state $\left(x_{2}, y_{2}\right)$, provided each corresponded to a state generated during the steps 
outlined above. To verify path independence one would need to conduct a similar process, now measuring $x$ for fixed $y$ values while varying $F_{x}$ (changing $F_{x}$ and $F_{y}$ by the same increments used above). This lab also provided students practice distinguishing between fixed $y$ and fixed $F_{y}$ processes and the relevance of each to particular measurements.

\section{CONNECTIONS TO THERMODYNAMICS}

The integrated lab was designed to allow students to reach the conclusion that there are two ways to manipulate the potential energy of the system, each corresponding to a particular force $\left(F_{x}\right.$ or $\left.F_{y}\right)$ and distance $(x$ or $y)$. The students were able to see the pairing of these quantities through the differential expression for work,

$$
d U=F_{x} d x+F_{y} d y
$$

which is a mechanical equivalent of the thermodynamic identity, using conjugate pairs of forces and distances rather than the canonical thermodynamic conjugate pairs: temperature and entropy, pressure and volume.

The instructor then introduced the concept of the potential energy of the system, $U$, as a state function determined by $x$ and $y$, a function whose total differential is given by:

$$
d U=\left(\frac{\partial U}{\partial x}\right)_{y} d x+\left(\frac{\partial U}{\partial y}\right)_{x} d y
$$

From Eqs. 1 and 2 the instructor extracted definitions for the forces as partial derivatives of the potential energy, a result which should be familiar from both classical mechanics and $\mathrm{E} \& \mathrm{M}$.

Using these expressions for $F_{x}$ and $F_{y}$ students were asked to express $\left(\frac{\partial F_{x}}{\partial y}\right)_{x}$ and $\left(\frac{\partial F_{y}}{\partial x}\right)_{y}$ as derivatives of U. From these new derivative expressions, and Clairaut's theorem (the order of mixed partials does not matter), students found a Maxwell Relation for their system that they could experimentally verify.

During the remaining three contact hours of the Interlude the instructor led students through additional mathematical techniques relevant to thermodynamics including partial derivative manipulations, the cyclic chain rule, and Legendre Transforms, using the physical parameters of the PDM. These exercises allowed students to practice these math methods and understand their results in the context of tangible experimental measurements.

\section{CONCLUSION}

We believe that the use of Partial Derivative Machines will help to build student understanding of partial derivatives by giving hands-on experiences that demonstrate the physical significance of partial derivatives. By presenting students with a mechanical exercise, students are given an accessible introduction to the mathematical techniques in thermodynamics that rely on the use of partial derivatives. Starting from prior physics knowledge and allowing students to explore the machine as we build towards mathematical concepts allows for a clearer introduction of the math before expecting students to work with less tangible physical systems.

As part of our ongoing efforts to reform the Energy and Entropy course, we plan to explore the affordances and constraints of this device more fully. In particular, we are interested in how the Partial Derivative Machine impacts student reasoning and attitudes about partial derivatives and thermodynamics and its effectiveness at getting students to connect classical and thermodynamic systems.

\section{ACKNOWLEDGMENTS}

This work was supported in part by NSF DUE-1023120.

\section{REFERENCES}

1. D. Meltzer, PERC Proceedings 790, 31-34 (2004).

2. J. Thompson, B. Bucy, and D. Mountcastle, PERC Proceedings 818, 77-80 (2005).

3. B. Bucy, J. Thompson, and D. Mountcastle, PERC Proceedings 883, 157-160 (2006).

4. D. Meltzer, Physics Education Research Conference 2009 1179, 31-34 (2009).

5. J. Thompson, C. Manogue, D. Roundy, and D. Mountcastle, PERC Proceedings 1413, 85-88 (2011).

6. D. Roundy, M. B. Kustusch, and C. Manogue, American Journal of Physics (2013), in press.

7. Paradigms in Physics: Course materials, physics.oregonstate.edu/portfolioswiki/courses:start.

8. C. A. Manogue, P. J. Siemens, J. Tate, K. Browne, M. L. Niess, and A. J. Wolfer, Am. J. Phys. 69, 978-990 (2001)

9. C. A. Manogue, and K. S. Krane, Phys. Today 56, 53-58 (2003).

10. J. F. Wagner, C. A. Manogue, and J. R. Thompson, AIP Conference Proceedings 1413, 89-92 (2011).

11. C. A. Manogue, D. Roundy, M. B. Kustusch, E. van Zee, T. Dray, A. Gupta, and J. Wagner, PERC Proceedings (2013, this volume).

12. D. Roundy, and M. Rogers, American Journal of Physics 81, 20-23 (2013). 